\title{
BMJ Open Helicobacter pylori eradication treatment for gastric carcinoma prevention in asymptomatic or dyspeptic adults: systematic review and Bayesian meta-analysis of randomised controlled trials
}

\author{
Teruhiko Terasawa, ${ }^{1}$ Chisato Hamashima, ${ }^{2}$ Katsuaki Kato, ${ }^{3}$ Isao Miyashiro, ${ }^{4}$ \\ Takaki Yoshikawa, ${ }^{5}$ Reo Takaku, ${ }^{6}$ Hiroshi Nishida ${ }^{7}$
}

To cite: Terasawa $\mathrm{T}$, Hamashima C, Kato K, et al. Helicobacter pylori eradication treatment for gastric carcinoma prevention in asymptomatic or dyspeptic adults: systematic review and Bayesian metaanalysis of randomised controlled trials. BMJ Open 2019;9:e026002. doi:10.1136/ bmjopen-2018-026002

- Prepublication history and additional material for this paper are available online. To view these files, please visit the journal online (http://dx.doi org/10.1136/bmjopen-2018026002).

Received 13 August 2018 Revised 29 July 2019

Accepted 02 September 2019

Check for updates

(C) Author(s) (or their employer(s)) 2019. Re-use permitted under CC BY-NC. No commercial re-use. See rights and permissions. Published by BMJ.

For numbered affiliations see end of article.

Correspondence to Dr Teruhiko Terasawa; terasawa@fujita-hu.ac.jp

\section{ABSTRACT}

Objectives Recent meta-analyses of eradication therapy in Helicobacter pylori-infected adults reported significant reductions in gastric carcinoma risk. However, concerns about supporting unfocused screening and eradication programme in healthy, asymptomatic populations have arisen. We performed a systematic review and Bayesian meta-analysis to provide an accurate interpretation of randomised evidence on the preventive effectiveness of eradication therapy on gastric carcinoma risk.

Methods We searched databases including PubMed, Cochrane Central and Embase for reference and citation tracking without language restrictions, from inception through 31 July 2018. Paired investigators independently selected randomised controlled trials (RCTs) comparing eradication therapy with placebo or no treatment for asymptomatic or dyspeptic $H$. pylori-infected adults with no previous gastric carcinoma. The main outcome was gastric carcinoma incidence; secondary outcomes included gastric carcinoma-specific, non-gastric carcinoma and all-cause mortality.

Results A total of 5 population-based and 2 outpatient care-based RCTs involving 7303 adults were eligible. Eradication algorithms were heterogeneous, and unsuccessful eradication and reinfection were frequently observed. A Bayesian meta-analysis with competing risk outcomes found low-certainty evidence that eradication therapy might be more likely than control to reduce gastric carcinoma risk (HR=0.65; 95\% credible interval (Crl) 0.41 to $1.0 ; P^{2}=11 \%$ ). The Crls included the null effects across the subgroup and sensitivity analyses, apart from those based on particular models that excluded two RCTs that enrolled subjects with specific histological findings only ( $\mathrm{HR}=0.55$; $\mathrm{Crl} 0.30$ to $0.89 ; P^{2}=14 \%$ ). The uncertainty of the average $41 \%$ risk reduction in gastric carcinomaspecific mortality included a clinically important mortality risk increase ( $\mathrm{HR}=0.59$ favouring eradication therapy; $\mathrm{Crl}$ 0.25 to $1.20 ; P^{2}=13 \%$; low certainty).

Conclusions There is insufficient evidence to support or refute the effectiveness of eradication therapy in preventing gastric carcinoma in H. pylori-infected, high-
Strengths and limitations of this study

- This is the first meta-analysis of the preventive effectiveness of eradication attempts in healthy-appearing Helicobacter pylori-infected subjects with no previous gastric carcinoma that adopted fully Bayesian approaches to account for multiple timeto-event outcomes with competing risks and frequent censored observations.

- This review also performed comprehensive literature searches, obtained updated data from the trial authors and used the Grading of Recommendation Assessment, Development and Evaluation approach to formally assess the certainty of the evidence.

- The eligible trials included subjects with high-risk preneoplastic lesions and adopted suboptimal eradication algorithms, which precluded reliable assessments of the true efficacy of successful eradication in healthy $H$. pylori-infected adults.

risk populations. Rigorously conducted large RCTs of healthy infected adults only would provide evidence of the true efficacy of successful eradication.

PROSPERO registration number: CRD42014009245.

\section{BACKGROUND}

Gastric carcinoma is the second most common cancer-specific cause of death worldwide and the third most prevalent cancer in East Asia. ${ }^{1}$ Infection with Helicobacter pylori is a strong risk factor for gastric carcinoma. ${ }^{2}$ Several postulated oncogenic mechanisms ${ }^{3}$ and epidemiological evidence ${ }^{45}$ supporting this association exist. Explanations for the high gastric carcinoma incidence in East Asia include environmental factors such as high sodium intake ${ }^{6}$ and the geographically distinct predominance of $H$. pylori strains 
characterised by strain-specific polymorphisms in the $\operatorname{cag} A$ gene, producing a highly virulent CagA. ${ }^{67}$

Since the establishment of effective eradication treatments in the 1990s, several randomised controlled trials (RCTs) have assessed whether eradicating H. pylori can reduce the risk of gastric carcinoma in healthy adults. After multiple reports of inconclusive results, with the long-term follow-up of the largest RCT that showed a significant reduction in gastric carcinoma risk in the eradication group, ${ }^{8}$ several meta-analyses that included RCTs only, ${ }^{9}{ }^{10}$ observational studies only, ${ }^{11}$ and both RCTs and observational studies ${ }^{12}$ reported similar findings. On this basis, clinical guidelines and consensus reports have consistently recommended screening and eradication of $H$. pylori in high-risk populations. ${ }^{13-18}$ However, concerns about implementing unfocused screening and eradication programme in healthy, asymptomatic populations still remain because these meta-analyses had several limitations. ${ }^{9-12}$ The evidence partly derived from studies focusing on patients treated for early-stage gastric carcinoma ${ }^{1011}$ cannot be directly applied to the general population. Also, concerns about the evidence obtained from cohort studies ${ }^{11} 12$ include confounding and other sources of bias. ${ }^{19}{ }^{20}$ A recent Cochrane review, the only meta-analysis focusing on RCTs of subjects without previous gastric carcinoma, ${ }^{9}$ relied on data reported in conference abstracts $^{21}$ and used statistical approaches that can calculate falsely narrow CIs when the number of studies is small and events are rare. ${ }^{22-25}$ Additionally, the sensitivity analyses used for exploring the effect of censored cases on the summary estimates were for binary outcomes, ${ }^{26}{ }^{27}$ not for time-to-event outcomes with competing risks. ${ }^{28}$

Bayesian random-effect meta-analysis is an increasingly used flexible methodology that accounts for uncertainty by combining all available external evidence as the initial belief through the likelihood function, using new data to update the evidence. ${ }^{29}{ }^{30}$ We conducted a systematic review and Bayesian meta-analysis to provide an accurate interpretation of the currently available evidence from RCTs on the preventive effect of $H$. pylori eradication on gastric carcinoma.

\section{METHODS}

This study is an updated systematic review and meta-analysis based on an evidence report that was produced for developing the Japanese Guidelines for Gastric Cancer Screening (see online supplementary methods for details). ${ }^{31}$

\section{Data sources and searches}

We searched PubMed, the Cochrane Central Register of Controlled Trials and Embase from inception through 31 July 2018 using search terms including "gastric cancer", "Helicobacter pylor", "eradication" and their synonyms, without language restrictions. To supplement the search, we perused the reference lists of eligible studies and relevant review articles. We also examined the titles and abstracts of all articles citing at least one of the publications already included, found through the citation-tracking functions of Web of Science, Scopus and Google Scholar (see online supplementary methods).

\section{Study selection}

Paired reviewers, drawn from the group of seven investigators (TT, CH, KK, IM, TY, RT, and HN), independently screened non-overlapping sets of abstracts and full texts of potentially eligible studies. RCTs that compared eradication therapies for $H$. pylori with placebo or no treatment in asymptomatic or otherwise healthy, dyspeptic $H$. pylori-infected adults (aged $\geq 18$ years) with no history of gastric carcinoma were eligible. We allowed for the inclusion of 'otherwise healthy, dyspeptic subjects' post hoc because both uninvestigated and functional dyspepsia are common in Asia, ${ }^{32}$ and the relevant RCTs had included such subjects. Trials reporting no gastric carcinoma events were excluded. We included trials published as full text only. Disagreements were resolved by consensus. Full details of the inclusion criteria are described in the online supplementary methods.

\section{Data extraction}

One reviewer (TT) extracted trial, subject and intervention characteristics from each eligible paper; another (KK) verified the data. If the paper with the longest follow-up period did not report the relevant information but cited earlier publications, we extracted data from the cited publications (see online supplementary methods).

Our primary outcome was the incidence of gastric carcinoma; non-carcinoma tumours (eg, lymphomas or sarcomas) were excluded. Our secondary outcomes were gastric carcinoma-specific mortality, non-gastric carcinoma mortality and all-cause mortality. Two reviewers (TT, IM) independently extracted the number of randomly allocated participants as the intention-to-treat analysis population, excluding any mistakenly enrolled ineligible subjects ${ }^{26} 33$ as well as the numbers of gastric carcinoma events, deaths from gastric carcinoma and other causes, and censored subjects as missing outcome data (MOD) for each arm from the publications with the longest follow-up. Censored subjects included those lost to follow-up for interim exclusion due to protocol deviation, non-compliance and withdrawal. ${ }^{34}$ Disagreements were resolved by consensus. For any missing information or unresolved discrepancies, we contacted the authors of the primary studies for clarification or to request unpublished data. We considered the request rejected if two emails received no response.

\section{Assessment of risk of bias}

Two independent reviewers (TT, TY) analysed sequence generation; allocation concealment; blinding of participants; trial personnel and outcome assessment; incomplete outcome data; selective reporting and other biases using the Cochrane Collaboration's Risk of Bias tool. ${ }^{35}$ The kappa coefficient for inter-rater agreement was 
0.76 , indicating good agreement. ${ }^{36}$ Disagreements were resolved by consensus.

\section{Data synthesis}

We calculated summary HRs and their 95\% credible intervals (CrIs) and prediction intervals (PrIs), ${ }^{30} 37$ using a hierarchical Bayesian random-effect meta-analysis method for competing risk time-to-event outcomes. ${ }^{38} 39$ For gastric carcinoma incidence, we modelled the baseline and relative hazard rates for gastric carcinoma events, non-gastric carcinoma deaths and MOD as mutually exclusive outcomes and their respective random-effect parameters in a single analysis; we assumed that no cases of gastric carcinoma later died from a cause other than gastric carcinoma. For analysing gastric carcinoma-specific and non-gastric carcinoma mortality, deaths from gastric carcinoma, deaths from all other causes and MOD were simultaneously modelled as mutually exclusive outcomes with their respective random-effect parameters. As a sensitivity analysis, we calculated summary ORs for cumulative gastric carcinoma incidence as a binary outcome. For MOD and non-gastric carcinoma deaths, we applied hierarchical Bayesian random-effect meta-analytic models using the conventional imputation methods for binary outcomes, ${ }^{26}$ and models directly allowing for uncertainty due to missing data. ${ }^{40}$

After conducting the analyses based on three alternative prior distributions on the between-trial standard deviations (tau), we selected the context-specific informative prior distributions in the main analysis. ${ }^{41}$ We determined this post-hoc selection since the data from only seven RCTs were deemed to be insufficient to inform multiple between-trial variance parameters using the other two conventional, less informative priors. ${ }^{24} 39$ For each model in the Bayesian meta-analysis, we based results on 3 different chains and 100000 iterations following a burn-in of 100000 iterations. We considered nodes to have converged when the Brooks-Gelman-Rubin statistic was $<1.010 .^{24}$ The online supplementary methods provide complete details of the methodologies, model fitting, choice of prior distributions for the parameters and the operational definitions used in the sensitivity analysis.

We assessed the certainty of evidence for each outcome using the Grading of Recommendation Assessment, Development and Evaluation approach. ${ }^{42}$ We quantified between-study heterogeneity using the tau and $I^{2}$ statistics with their $95 \%$ CrIs. ${ }^{43}$ To examine how each trial affected the summary estimate of the other RCTs, we performed a leave-one-out meta-analysis by calculating summary estimates iteratively, excluding one trial at a time. ${ }^{44}$ We did not perform the planned tests for funnel plot asymmetry because eligible studies were $<10 .{ }^{45}$ To explore clinical heterogeneity, we performed trial-level subgroup analyses and univariable meta-regressions according to geographic location (East Asia vs other countries), research setting (community vs outpatient) and subject selection (all invited subjects regardless of baseline gastric histology vs those with specific histological subtypes). ${ }^{44} 46$ All analyses were conducted using Stata/SE V.14.2 (Stata Corp) and WinBUGS 1.4.3 (MRC Biostatistics Unit, Cambridge, UK). P values for all comparisons were two tailed, and statistical significance was defined as $\mathrm{p}<0.05$.

\section{Patient and public involvement}

Although this study had no direct patient involvement, we modified the systematic review protocol based on input and feedback from the public regarding draft guidelines containing a set of interim results. ${ }^{31}$

\section{RESULTS}

\section{Literature search and eligible studies}

After abstract-level screening of the main searches, citation index searches and citations identified from other sources, we reviewed the full texts of 42 potentially eligible published articles (online supplementary figure 1). After exclusions and review of unpublished data provided by the authors, 7 independent RCTs reported in 16 publications were ultimately eligible. ${ }^{847-61}$ We excluded the longterm follow-up reports from one $\mathrm{RCT}^{62} 63$ because of a possibility of treatment contamination due to crossover (64\% of participants in the control group received eradication therapy after 6 years of follow-up). The online supplementary results provide full details of the study selection and excluded studies.

\section{Study and clinical characteristics}

The seven RCTs-five from China, 847555661 one from Japan, ${ }^{60}$ and one from Colombia ${ }^{58}$-included 7303 H. pylori-infected adults without gastric carcinoma, as confirmed by upper endoscopic examination (online supplementary table 1). Two population-based RCTs ${ }^{4755}$ and one outpatient care trial ${ }^{60}$ explicitly included participants with dyspeptic symptoms in addition to asymptomatic subjects. The other trials, three population-based ${ }^{85658}$ and one outpatient care-based RCT, ${ }^{61}$ did not report on symptoms at enrolment. One RCT diagnosed $H$. pylori infection by serological testing only. ${ }^{8}$ Four trials used a parallel design, ${ }^{47556061}$ whereas the other three adopted factorial designs with cointerventions of vitamins ${ }^{58}$; vitamins, selenium and garlic extract ${ }^{8}$; or celecoxib, a cyclooxygenase-2 inhibitor. ${ }^{56}$ Two RCTs focused on populations with specific precancerous lesions based on pre-enrolment histology results. ${ }^{56}$ The follow-up duration extended beyond 10 years in only two trials. ${ }^{85}$

Gastric carcinoma incidence was the primary outcome in only one $\mathrm{RCT}^{47}$ Another long-term follow-up report from the largest trial cohort reported gastric carcinoma as the post hoc primary outcome. ${ }^{8}$ No trials defined gastric carcinoma-specific mortality or all-cause mortality as their primary outcomes before conducting the study. Methods used to confirm the outcomes were non-uniform. Four RCTs periodically performed endoscopic gastric surveys with biopsies of multiple sites ${ }^{855860}$; the other three scheduled similar routine gastric surveys only once. ${ }^{47561}$ Three trials also examined patients' clinical records, the 
regional cancer registry and/or death certificates to identify gastric carcinoma cases and death events. ${ }^{84756}$

The sample sizes ranged from 236 to 2258 (median, 852 ) subjects with an average age of $42-53$ years (online supplementary table 2). Precancerous gastric lesions at enrolment varied across trials; one trial that enrolled subjects with advanced gastric lesions reported gastric dysplasia in $54 \%$ of the participants. ${ }^{56}$ The adopted eradication algorithms also varied. Two trials repeated first-line therapy for non-responders, ${ }^{860}$ two provided no salvage therapy ${ }^{556}$ and one employed dual combination therapy. 8 While the post-treatment eradication rates assessed at 1-72 months ranged from $72 \%$ to $89 \%$, follow-up eradication rates at 7-12 years were approximately $50 \%$, suggesting recurrence or reinfection. ${ }^{8562}$ The baseline incidence of gastric carcinoma ranged from 107 to 1802 per 100000 person years. The two RCTs of populations with specific histological findings reported the lowest and second lowest incident rates. ${ }^{568}$ Gastric carcinoma events were missing in $565(15 \%)$ and $543(15 \%)$ participants in the eradication and control arms, respectively.

\section{Assessment of risk of bias}

Overall, only one RCT, a long-term follow-up report of the largest trial, ${ }^{8}$ was deemed to have a low risk of bias (online supplementary figure 2 and table 3). Only two RCTs used the proportional hazard model to analyse time-to-event outcomes-one for gastric carcinoma incidence, ${ }^{56}$ the other for mortality. ${ }^{8}$

\section{Incidence of gastric carcinoma}

A total of 7 RCTs, including 7303 adults, observed 138 gastric carcinoma events (table 1). They failed to observe the primary outcome in 369 subjects who died from non-gastric carcinoma causes and in 649 subjects with MOD. Although the summary estimates showed a $35 \%$ risk reduction for gastric carcinoma associated with eradication therapy, the upper limit of the $95 \%$ CrI included 1 , the null effect, suggesting inconclusive average preventive effects of eradication therapy on gastric carcinoma risk (HR $=0.65$ (95\% CrI 0.41 to $1.0 ; 95 \% \operatorname{PrI} 0.30$ to 1.33 ); $I^{2}=11 \%$ (95\% CrI, $1 \%$ to $\left.54 \%\right)$; tau $=0.21$; low certainty) (figure 1). The certainty of the evidence was downrated because of imprecision due to the small number of events and limited clinical applicability in current practice due to unsatisfactory eradication strategies and frequent reinfection. Wide CrIs for $I^{2}$ and tau also suggested the possibility of statistical heterogeneity. The online supplementary results provide detailed descriptions of the model fitting.

\section{Gastric carcinoma-specific mortality}

A total of 3 community-based RCTs (4400 adults) provided data on deaths from gastric carcinoma (table 1). Although gastric carcinoma-specific mortality was reduced on average by $41 \%$, its uncertainty included both clinically important mortality benefit and risk increases $(\mathrm{HR}=0.59$ favouring eradication therapy (95\% CrI 0.25 to $1.20 ; 95 \%$ PrI 0.18 to 1.63 ); $I^{2}=13 \%$ (95\% CrI $1 \%$ to $66 \%$ ); $t a u=0.26$; low certainty) (figure 1). Concerns about precision due to the wide CrIs and indirectness due to the adoption of inadequate eradication strategies downgraded certainty.

\section{All-cause mortality and non-gastric carcinoma mortality}

A total of 5 community-based RCTs (6316 adults) contributed data on deaths from all and non-gastric carcinoma causes (table 1). Overall, there was no evidence that eradication therapy increased or decreased all-cause mortality ( $\mathrm{HR}=0.97$ favouring eradication therapy $(95 \% \mathrm{CrI} 0.69$ to 1.28 ; $95 \%$ PrI 0.55 to 1.56 ); $I^{2}=20 \%$ (95\% CrI $2 \%$ to $68 \%$ ); tau $=0.15$; low certainty) or mortality from causes other than gastric carcinoma ( $\mathrm{HR}=1.03$ favouring control $(95 \%$ CrI 0.71 to $1.41 ; 95 \%$ PrI 0.54 to 1.80 ); $I^{2}=21 \%$ (95\% CrI $1 \%$ to $76 \%$ ); tau=0.16; low certainty) (figure 1). Certainty was downrated because of the wide CrIs that included a clinically important mortality benefit and increase.

\section{Subgroup analysis}

The subgroup meta-analysis for trials conducted in East Asia found an average reduction in gastric carcinoma risk of $38 \%$ (six trials; HR $=0.62$ (95\% CrI 0.37 to $0.98 ; 95 \%$ PrI 0.27 to 1.33 ); $I^{2}=13 \%$ (95\% CrI $1 \%$ to $60 \%$ ); tau $=0.22$; relative $\mathrm{HR}=0.95$ (95\% CrI 0.19 to 6.57$)$ ). A higher risk reduction was suggested in the subgroup analysis in which the two trials of populations with specific histological findings were excluded (five trials; $\mathrm{HR}=0.55$ (95\% CrI 0.30 to $0.89 ; 95 \%$ PrI 0.22 to 1.19$) ; I^{2}=14 \%$ (95\% CrI $1 \%$ to $63 \%)$; tau $=0.22$; relative $\mathrm{HR}=0.54(95 \%$ CrI 0.15 to 1.62)) (online supplementary table 4 ). The metaregression analyses showed no differential effects by geographic location, research setting or subject selection.

\section{Sensitivity analysis}

For gastric carcinoma incidence, the CrIs for the summary HRs generally became wider, with the upper limit crossing 1, when alternative, less informative priors for tau were specified except for the subgroup analysis where the two trials of populations with specific histological findings were excluded (online supplementary table 4). Similarly, the CrIs crossed 1, unless 1 of 2 RCTs for histologically selected populations was excluded in the leave-one-out meta-analysis (online supplementary figure 3 ). In the sensitivity analyses using the conventional Bayesian meta-analysis for binary outcomes, the summary ORs were generally congruent to the summary HRs of the main analysis. The CrIs were generally wide and crossed the null effects (ie, OR=1) regardless of the prior distributions for tau, the use of alternative imputation methods or models directly accounting for uncertainty to account for MOD, or exclusion of non-gastric carcinoma deaths from MOD, except for the subgroup analysis where two trials of histologically selected populations were excluded (online supplementary table 5). The summary HRs for the mortality outcomes were not materially different from the results in the main analysis when alternative priors for tau were used, or all seven trials were included in the sensitivity analysis (online supplementary table 4 ). 


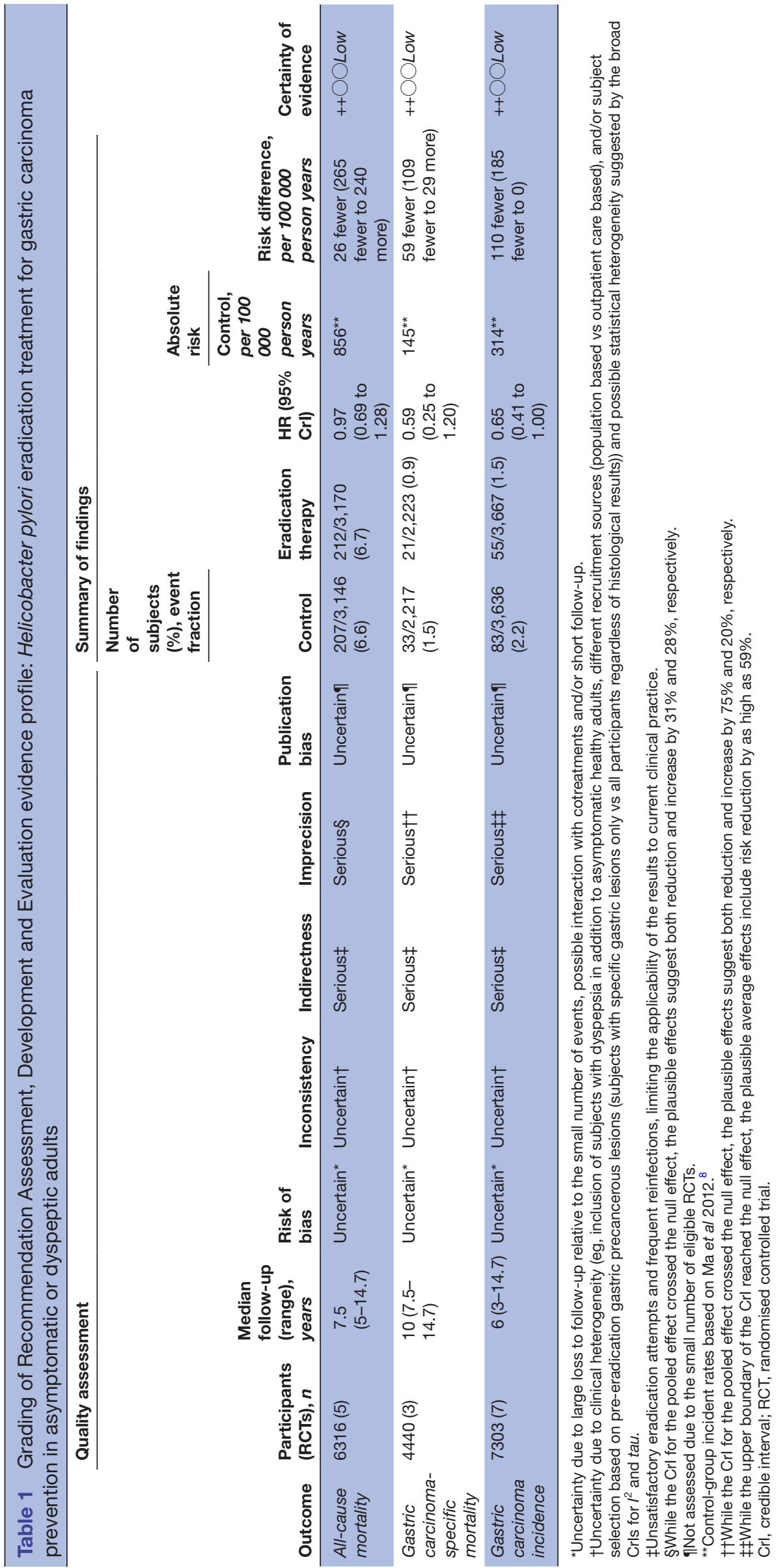




\begin{tabular}{|c|c|c|c|c|}
\hline Trial ID & $\begin{array}{l}\text { Crude HR (Cl) } \\
\text { Summary HR (Crl [Prl]) }\end{array}$ & Adjusted HR (Crl) & $\begin{array}{l}\text { Eradication, } \\
\mathrm{n} / \mathrm{n}\end{array}$ & $\begin{array}{l}\text { Control, } \\
\mathrm{n} / \mathrm{n}\end{array}$ \\
\hline \multicolumn{5}{|l|}{ Gastric carcinoma incidence } \\
\hline Correa et al. $2000^{58}$ & $1.43(0.24$ to 8.53$)$ & $0.65(0.34$ to 1.21$)$ & $3 / 437$ & $2 / 415$ \\
\hline Wong et al. $2004^{47}$ & $0.63(0.24$ to 1.62$)$ & $0.63(0.34$ to 1.05$)$ & $7 / 817$ & $11 / 813$ \\
\hline Saito et al. $2006^{57}$ & $0.53(0.10$ to 2.90$)$ & $0.63(0.31$ to 1.14$)$ & $2 / 379$ & $4 / 372$ \\
\hline Tang et al. $2010^{81}$ & 0.23 (0.03 to 2.05 ) & 0.65 (0.31 to 1.25$)$ & $1 / 118$ & $4 / 118$ \\
\hline Ma et al. $2012^{8}$ & $0.65(0.42$ to 1.00$)$ & $0.67(0.45$ to 0.96$)$ & $34 / 1,130$ & $52 / 1,128$ \\
\hline Wong et al. $2012^{56}$ & 2.02 (0.51 to 8.08$)$ & $0.71(0.41$ to 1.44$)$ & $6 / 510$ & $3 / 514$ \\
\hline Zhou et al. $2014^{55}$ & 0.28 (0.06 to 1.36$)$ & $0.60(0.28$ to 1.04$)$ & 2/276 & $7 / 276$ \\
\hline Summary; $F^{2}=11 \%($ Crl: 1 to $54 \%)$ & 0.65 (0.41 to $1.00[0.30$ & to 1.33$]$ ) & $55 / 3,667$ & $83 / 3,636$ \\
\hline \multicolumn{5}{|l|}{ Gastric carcinoma-specific mortality } \\
\hline Wong et al. $2004^{47}$ & $0.50(0.12$ to 1.99$)$ & $0.56(0.22$ to 1.17$)$ & $3 / 817$ & $6 / 813$ \\
\hline Ma et al. $2012^{8}$ & $0.65(0.35$ to 1.20$)$ & $0.66(0.37$ to 1.16$)$ & $17 / 1,130$ & $24 / 1,128$ \\
\hline Zhou et al. $2014^{55}$ & $0.33(0.04$ to 3.21$)$ & $0.56(0.18$ to 1.25$)$ & $1 / 276$ & $3 / 276$ \\
\hline Summary; $I^{2}=13 \%$ (Crl: 1 to $\left.66 \%\right)$ & $0.59(0.25$ to $1.20[0.18$ & to 1.63$])$ & $21 / 2,223$ & $33 / 2,217$ \\
\hline \multicolumn{5}{|l|}{ Non-gastric carcinoma mortality } \\
\hline Correa et al. $2000^{58}$ & $1.90(0.72$ to 5.01$)$ & $1.10(0.73$ to 1.90$)$ & $12 / 437$ & $6 / 415$ \\
\hline Wong et al. $2004^{47}$ & 1.11 (0.58 to 2.12$)$ & 1.04 (0.68 to 1.53$)$ & $19 / 817$ & $17 / 813$ \\
\hline Ma et al. $2012^{\circ}$ & $1.18(0.94$ to 1.49$)$ & $1.13(0.91$ to 1.43$)$ & $140 / 1,130$ & $118 / 1,128$ \\
\hline Wong et al. $2012^{56}$ & 0.67 (0.19 to 2.37$)$ & $0.99(0.51$ to 1.50$)$ & $4 / 510$ & $6 / 514$ \\
\hline Zhou et al. $2014^{55}$ & $0.59(0.33$ to 1.08$)$ & $0.92(0.52$ to 1.29$)$ & $16 / 276$ & $27 / 276$ \\
\hline Summary; $l^{2}=21 \%$ (Crl: 1 to $\left.76 \%\right)$ & $1.03(0.71$ to $1.41[0.54$ & to 1.80$]$ ) & 191/3,170 & $174 / 3,146$ \\
\hline \multicolumn{5}{|l|}{ All-cause mortality } \\
\hline Correa et al. $2000^{58}$ & 1.90 (0.71 to 5.06$)$ & $1.03(0.69$ to 1.67$)$ & $12 / 437$ & $6 / 415$ \\
\hline Wong et al. $2004^{47}$ & 0.95 (0.53 to 1.71$)$ & $0.96(0.65$ to 1.35$)$ & $22 / 817$ & $23 / 813$ \\
\hline Ma et al. $2012^{8}$ & 1.14 (0.90 to 1.46$)$ & 1.06 (0.86 to 1.31$)$ & $157 / 1,130$ & $142 / 1,128$ \\
\hline Wong et al. $2012^{56}$ & 0.67 (0.19 to 2.38$)$ & 0.94 (0.53 to 1.38$)$ & $4 / 510$ & $6 / 514$ \\
\hline Zhou et al. $2014^{55}$ & 0.57 (0.31 to 1.03$)$ & 0.87 (0.52 to 1.21$)$ & $17 / 276$ & $30 / 276$ \\
\hline Summary; $F^{2}=20 \%($ Crl: 2 to $68 \%)$ & 0.97 (0.69 to $1.28[0.55$ & to 1.56$]$ ) & $212 / 3,170$ & $207 / 3,146$ \\
\hline
\end{tabular}

Figure 1 Effect of eradication therapy on gastric carcinoma incidence, gastric carcinoma-specific mortality, non-gastric carcinoma mortality, and all-cause mortality in asymptomatic or dyspeptic Helicobacter pylori-infected adults. Diamonds represent the summary HRs centred on a combined estimate and extending to $95 \%$ credible intervals (Crls), with estimated 95\% prediction intervals (Prls) depicted as horizontal lines. Black squares and horizontal lines indicate crude 'observed' HRs and $95 \%$ Cls. Grey squares and horizontal lines indicate 'adjusted' HRs and 95\% Crls based on the posterior distribution for individual studies. The size of the square is proportional to the inverse of the variance of the logarithm-transformed HR of each study. Studies are ordered by publication year. The colours of the Cls and Crls for non-gastric carcinoma mortality and all-cause mortality for the study by $\mathrm{Ma}$ et $\mathrm{al}^{8}$ are inverted. Prl, prediction interval. 


\section{DISCUSSION}

\section{Main findings}

Our meta-analysis found only a low strength of evidence that $H$. pylori eradication may have been more likely than control treatment to reduce gastric carcinoma development-although the average effect suggested a risk reduction by $30 \%$, its uncertainty included the null effects. This finding was generally consistent across subgroup and sensitivity analyses except when two factorial design trials that focused on enrolees with specific histological findings were excluded. Although we calculated a similar average effect size, our wide CrIs were not congruent with those of previous meta-analyses including the Cochrane meta-analysis that reported that $H$. pylori eradication significantly reduced gastric carcinoma risk. ${ }^{9}$ This highlights the uncertainty surrounding the effectiveness of unfocused test-and-eradicate programme, even in highrisk regions. A recently published fixed-effect meta-analysis using risk difference as the outcome measure, although naïvely conducted, reported a non-significant risk reduction associated with eradication therapy, which raised concerns about the conclusions from previous meta-analyses. ${ }^{64}$ The results of our Bayesian meta-analysis corroborate this finding.

The preventive effects became larger and significantan average of $45 \%$ risk reduction-when the two factorial design trials that selected more histologically advanced lesions were excluded. This suggests that eradication therapy may be more effective in non-focused, potentially lower risk populations; this is in line with the meta-analysis of a subgroup from the primary prevention RCTs conducted by Chen et al. ${ }^{10}$ The fixed-effect meta-analysis of subjects with better pre-eradication histology than intestinal metaplasia or dysplasia only, comprising only 1 case who developed gastric carcinoma out of 1221 subjects in the eradication group versus 10 cases out of 1228 in the control group, although the balance between the two groups created from randomisation might no longer hold, reported a significant $78 \%$ risk reduction (relative risk $=0.22(95 \%$ CI 0.06 to 0.86$))$. However, the severity of the baseline precancerous lesions across the trials could not directly be compared due to variations including the histological criteria used. Furthermore, in addition to baseline histology, other factors that affected gastric carcinoma events would exist, given the lowest baseline incidence rates in the two factorial RCTs of histologically 'high-risk' populations. For example, given the subjective nature of endoscopic evaluation in particular, 'missed' cases of gastric carcinoma at enrolment and/or during the follow-up rounds are always a concern affecting the validity of gastric carcinoma research. ${ }^{65}$ Therefore, our observation based on subgroup analysis should be viewed as hypothesis generation.

We found that evidence on gastric carcinoma-specific and overall mortality was insufficient because the RCTs were not powered to detect differences in these outcomes. The wide CrIs for the average effect suggest that while a clinically meaningful $75 \%$ reduction in gastric carcinoma-specific mortality is possible, a $20 \%$ increase cannot be ruled out.

Our meta-analysis has several strengths. We employed Bayesian approaches to account for multiple time-to-event outcomes with competing risks and frequent censored observations. Our literature search found one RCT ${ }^{61}$ that previous meta-analyses had failed to include. We also used data extracted from full-text publications and obtained unpublished data from two RCTs, ${ }^{5560}$ for which previous meta-analyses had relied on the data presented in conference abstracts ${ }^{9} 12$ or earlier short-term reports (online supplementary table 6 ) ${ }^{10}$ These additional data allowed us to perform a more comprehensive analysis than was possible in previous meta-analyses that focused on RCTs only ${ }^{9} 1064$ regarding across-trial differences in research settings, patient symptoms at enrolment, missing outcomes and mortality events.

\section{Limitations}

Limitations of the included RCTs other than short, incomplete follow-ups, need to be noted. The RCTs employed potentially suboptimal eradication algorithms and reported frequent rates of recurrence or reinfection. These unsuccessful eradication attempts could have affected the preventive effect. In addition, as already noted, inclusion of RCTs that allowed for the inclusion of subjects with advanced preneoplastic lesions, those at high risk for progression to gastric carcinoma, ${ }^{13}$ might have attenuated the preventive effects. Moreover, interactions with concurrent interventions, regardless of their directions, cannot be ruled out in the three factorial design trials. Similar to previous meta-analyses, ${ }^{9} 1064$ our Bayesian meta-analysis could not address these limitations analytically without access to individual-level data.

In the meta-analysis of gastric carcinoma incidence, our assumption that those who developed gastric carcinoma would not die from causes other than gastric carcinoma should be clinically acceptable. Nevertheless, more accurate analysis would require individual-level data or modelling with additional assumptions for the conditional risk of non-gastric carcinoma deaths.

\section{Implications}

Given the insufficient randomised evidence, rigorously conducted large RCTs adopting stringent research methodologies would be ideal if demonstration of the true efficacy of eradication therapy is the central issue. This context would include the use of more effective eradication strategies, including accurate response assessment, better salvage therapies and meticulous longer term follow-up. Implementation of high-quality pre-enrolment gastric survey, including the strict exclusion of subjects who already have preneoplastic lesions, would be particularly relevant if healthy subjects are truly targeted. Nevertheless, challenges exist when conducting de novo long-term large high-quality RCTs of rare outcomes. Hence, the analysis of the current trends using large, clinical, practice-based registries is a realistic primary research 
option. If feasible, conducting individual-level consortia meta-analysis of all existing RCTs remains a viable option to repurpose the already available resources. Exploring effect modifiers for identifying subgroups that benefit most (or least) from the eradication strategy should be the highest priority. ${ }^{37}$ Further, we should not ignore all-cause and gastric carcinoma-specific mortality and long-term adverse events as important outcomes attributable to eradication.

In conclusion, there is insufficient randomised evidence to support or refute the effectiveness of eradication therapy in preventing gastric carcinoma in $H$. pylori-infected, high-risk populations. Large RCTs of only healthy $H$. pylori-infected adults adopting more stringent research methodologies will provide evidence regarding the true efficacy of successful eradication.

\section{Author affiliations \\ ${ }^{1}$ Emergency and General Internal Medicine, Fujita Health University, Toyoake, Japan ${ }^{2}$ Department of Nursing, Faculty of Medical Technology, Teikyo University, Tokyo, Japan \\ ${ }^{3}$ Cancer Detection Center, Miyagi Cancer Society, Sendai, Japan \\ ${ }^{4}$ Department of Cancer Registration and Survey, Osaka Medical Center for Cancer and Cardiovascular Diseases, Osaka, Japan \\ ${ }^{5}$ Department of Gastrointestinal Surgery, Kanagawa Cancer Center, Yokohama, Japan \\ ${ }^{6}$ Institute for Health Economics and Policy, Tokyo, Japan \\ ${ }^{7}$ AMS New Otani Clinic, Osaka, Japan}

Acknowledgements The authors thank Daizo Saito, Liya Zhou and Rongli Cui for providing unpublished data from their original studies; Alexander Ford for providing additional information regarding the data extraction processes used for the Cochrane meta-analysis; Yujia Cui-Tsuzuki for translating the Chinese-language publications; and Christopher Schmid for assisting with the statistical analysis.

Contributors TT and $\mathrm{CH}$ conceived the initial idea, performed the literature search and are the guarantors for the study. All authors designed the study, determined the eligibility of primary studies, acquired the data, interpreted the findings and read and approved the final manuscript. TT performed the statistical analyses and drafted the first version of the report, which was critically revised by all authors.

Funding This work was supported by the Ministry of Health, Labour and Welfare, Japan. TT and $\mathrm{CH}$ were supported in part by the Ministry of Education, Culture, Sports, Science and Technology, Japan (NNo. 26460755).

Disclaimer The funding sources had no role in the design or conduct of the study; collection, management, analysis and interpretation of the data; preparation, review or approval of the manuscript; or the decision to submit the manuscript for publication.

Competing interests All authors declare no conflicts of interest associated with this publication. For complete transparency, TY reports taking grants and personal fees from Chugai Pharmaceutical Co., grants and personal fees from Taiho Pharmaceutical, grants and personal fees from Yakult, personal fees from Lilly, grants from Novartis, personal fees from Ono, personal fees from Takeda, personal fees from Nippon Kayaku Co., personal fees from Covidien, personal fees from Johnson and Johnson, personal fees from Olympus, personal fees from NPO Epidemiological and Clinical Research Information Network, personal fees from NPO KSATTS, and personal fees from NPO Cancer Net Japan, outside the submitted work.

Patient consent for publication Not required.

Provenance and peer review Not commissioned; externally peer reviewed.

Data availability statement All data relevant to the study are included in the article or uploaded as supplementary information.

Open access This is an open access article distributed in accordance with the Creative Commons Attribution Non Commercial (CC BY-NC 4.0) license, which permits others to distribute, remix, adapt, build upon this work non-commercially, and license their derivative works on different terms, provided the original work is properly cited, appropriate credit is given, any changes made indicated, and the use is non-commercial. See: http://creativecommons.org/licenses/by-nc/4.0/.

\section{REFERENCES}

1. Fitzmaurice C, Akinyemiju TF, Al Lami FH, et al. Global, regional, and National cancer incidence, mortality, years of life lost, years lived with disability, and Disability-Adjusted life-years for 29 cancer groups, 1990 to 2016: a systematic analysis for the global burden of disease study. JAMA Oncol 2018;4:1553-68.

2. IARC Working Group on the Evaluation of Carcinogenic Risks to Humans. Biological agents. volume $100 \mathrm{~B}$. A review of human carcinogens. IARC Monogr Eval Carcinog Risks Hum 2012;100:1-441.

3. Polk DB, Peek RM. Helicobacter pylori: gastric cancer and beyond. Nat Rev Cancer 2010;10:403-14.

4. Helicobacter and Cancer Collaborative Group. Gastric cancer and Helicobacter pylori: a combined analysis of 12 case control studies nested within prospective cohorts. Gut 2001:49:347-53

5. Terasawa T, Nishida $\mathrm{H}$, Kato K, et al. Prediction of gastric cancer development by serum pepsinogen test and Helicobacter pylori seropositivity in eastern Asians: a systematic review and metaanalysis. PLoS One 2014;9:e109783.

6. Karimi P, Islami F, Anandasabapathy S, et al. Gastric cancer: descriptive epidemiology, risk factors, screening, and prevention. Cancer Epidemiology Biomarkers \& Prevention 2014;23:700-13.

7. Bridge DR, Merrell DS. Polymorphism in the Helicobacter pylori CagA and vacA toxins and disease. Gut Microbes 2013;4:101-17.

8. Ma J-L, Zhang L, Brown LM, et al. Fifteen-year effects of Helicobacter pylori, garlic, and vitamin treatments on gastric cancer incidence and mortality. J Natl Cancer Inst 2012;104:488-92.

9. Ford AC, Forman D, Hunt RH, et al. Helicobacter pylori eradication therapy to prevent gastric cancer in healthy asymptomatic infected individuals: systematic review and meta-analysis of randomised controlled trials. BMJ 2014;348:g3174.

10. Chen H-N, Wang Z, Li X, et al. Helicobacter pylori eradication cannot reduce the risk of gastric cancer in patients with intestinal metaplasia and dysplasia: evidence from a meta-analysis. Gastric Cancer 2016;19:166-75.

11. Doorakkers $E$, Lagergren J, Engstrand L, et al. Eradication of Helicobacter pylori and Gastric Cancer: A Systematic Review and Meta-analysis of Cohort Studies. J Natl Cancer Inst 2016;108:djw132.

12. Lee Y-C, Chiang T-H, Chou C-K, et al. Association between Helicobacter pylori eradication and gastric cancer incidence: a systematic review and meta-analysis. Gastroenterology 2016;150:1113-24.

13. Sugano K, Tack J, Kuipers EJ, et al. Kyoto global consensus report on Helicobacter pylori gastritis. Gut 2015;64:1353-67.

14. The committee of the Japanese society for Helicobacter research. Guidelines for diagnosis and treatment of $\mathrm{H}$. pylori infection in Japan: 2016 revised edition. Tokyo Sentan Igaku-Sha; 2016.

15. Malfertheiner P, Megraud F, O'Morain CA, et al. Management of Helicobacter pylori infection-the Maastricht V/Florence consensus report. Gut 2017;66:6-30.

16. Fock KM, Talley N, Moayyedi P, et al. Asia-Pacific consensus guidelines on gastric cancer prevention. $J$ Gastroenterol Hepatol 2008;23:351-65.

17. Liu WZ, Xie $\mathrm{Y}$, Cheng $\mathrm{H}$, et al. Fourth Chinese national consensus report on the management of Helicobacter pylori infection. J Dig Dis 2013;14:211-21.

18. Kim SG, Jung H-K, Lee HL, et al. Guidelines for the diagnosis and treatment of Helicobacter pylori infection in Korea, 2013 revised edition. J Gastroenterol Hepatol 2014;29:1371-86.

19. Sterne JAC, Hernán MA, Reeves BC, et al. ROBINS-I: a tool for assessing risk of bias in non-randomised studies of interventions. BMJ 2016;355.

20. Collins R, Reith C, Emberson J, et al. Interpretation of the evidence for the efficacy and safety of statin therapy. The Lancet 2016;388:2532-61.

21. Falagas ME, Rosmarakis ES. Clinical decision-making based on findings presented in conference Abstracts: is it safe for our patients? Eur Heart J 2006;27:2038-9.

22. Cornell JE, Mulrow CD, Localio R, et al. Random-effects metaanalysis of inconsistent effects: a time for change. Ann Intern Med 2014;160:267-70.

23. Fu R, Gartlehner G, Grant M, et al. Conducting quantitative synthesis when comparing medical interventions: AHRQ and the effective health care program. J Clin Epidemiol 2011;64:1187-97. 
24. Welton NJ, Sutton AJ, Cooper NJ, et al. Evidence synthesis for decision making in healthcare. Hoboken: John Wiley \& Sons Ltd, 2012.

25. Guolo A, Varin C. Random-effects meta-analysis: the number of studies matters. Stat Methods Med Res 2017;26:1500-18.

26. Akl EA, Johnston BC, Alonso-Coello P, et al. Addressing dichotomous data for participants excluded from trial analysis: a guide for systematic reviewers. PLoS One 2013;8:e57132.

27. Akl EA, Kahale LA, Agoritsas T, et al. Handling trial participants with missing outcome data when conducting a meta-analysis: a systematic survey of proposed approaches. Syst Rev 2015;4:98.

28. Koller MT, Raatz H, Steyerberg EW, et al. Competing risks and the clinical community: irrelevance or ignorance? Stat Med 2012;31:1089-97.

29. Welton NJ, Sutton AJ, Cooper NJ, et al. Introduction to decision models. in: evidence synthesis for decision making in healthcare. Hoboken: John Wiley \& Sons Ltd 2012:43-75.

30. Higgins JPT, Thompson SG, Spiegelhalter DJ. A re-evaluation of random-effects meta-analysis. J $R$ Stat Soc Ser A Stat Soc 2009;172:137-59.

31. The Steering Committee for the Development of Gastric Cancer Screening Guidelines. [The Japanese Guidelines for Gastric Cancer Screening 2014]. Available: http://canscreen.ncc.go.jp/guideline/ igan.html [Accessed 1 Mar 2018].

32. Ghoshal UC, Singh R, Chang F-Y, et al. Epidemiology of uninvestigated and functional dyspepsia in Asia: facts and fiction. $J$ Neurogastroenterol Motil 2011;17:235-44.

33. Fergusson D, Aaron SD, Guyatt G, et al. Post-Randomisation exclusions: the intention to treat principle and excluding patients from analysis. BMJ 2002;325:652-4.

34. Higgins JPT, White IR, Wood AM. Imputation methods for missing outcome data in meta-analysis of clinical trials. Clin Trials 2008;5:225-39.

35. Higgins JPT, Altman DG, Gøtzsche PC, et al. The Cochrane collaboration's tool for assessing risk of bias in randomised trials. BMJ 2011;343:d5928.

36. Altman DG. Practical statistics for medical research. Taylor \& Francis, 1990.

37. Riley RD, Higgins JPT, Deeks JJ. Interpretation of random effects meta-analyses. BMJ 2011;342:d549.

38. Ades AE, Mavranezouli I, Dias S, et al. Network meta-analysis with competing risk outcomes. Value Health 2010;13:976-83.

39. Dias S, Welton NJ, Sutton AJ. NICE DSU technical support document 2: a generalised linear modelling framework for pairwise and network meta-analysis of randomised controlled trials. 2011, 2016. Available: https://scharr.dept.shef.ac.uk/nicedsu/technicalsupport-documents/evidence-synthesis-tsd-series/

40. Turner NL, Dias S, Ades AE, et al. A Bayesian framework to account for uncertainty due to missing binary outcome data in pairwise metaanalysis. Stat Med 2015;34:2062-80.

41. Turner RM, Jackson D, Wei Y, et al. Predictive distributions for between-study heterogeneity and simple methods for their application in Bayesian meta-analysis. Stat Med 2015;34:984-98.

42. Guyatt GH, Oxman AD, Vist GE, et al. GRADE: an emerging consensus on rating quality of evidence and strength of recommendations. BMJ 2008;336:924-6.

43. Higgins JPT, Thompson SG. Quantifying heterogeneity in a metaanalysis. Stat Med 2002;21:1539-58.

44. Gagnier JJ, Morgenstern H, Altman DG, et al. Consensus-Based recommendations for investigating clinical heterogeneity in systematic reviews. BMC Med Res Methodol 2013;13:106.

45. Sterne JAC, Sutton AJ, loannidis JPA, et al. Recommendations for examining and interpreting funnel plot asymmetry in meta-analyses of randomised controlled trials. BMJ 2011;343:d4002.

46. Pocock S, Calvo G, Marrugat J, et al. International differences in treatment effect: do they really exist and why? Eur Heart $J$ 2013;34:1846-52.
47. Wong BC-Y, Lam SK, Wong WM, et al. Helicobacter pylori eradication to prevent gastric cancer in a high-risk region of China: a randomized controlled trial. JAMA 2004;291:187-94.

48. Gail MH, Brown LM, YouWC. Re: chemoprevention of gastric dysplasia: randomized trial of antioxidant supplements and antiHelicobacter pylori therapy. J Natl Cancer Inst 2001;93:559-60.

49. Gail MH, You WC, Chang YS, et al. Factorial trial of three interventions to reduce the progression of precancerous gastric lesions in Shandong, China: design issues and initial data. Control Clin Trials 1998;19:352-69.

50. You W-cheng, Brown LM, Zhang L, et al. Randomized doubleblind factorial trial of three treatments to reduce the prevalence of precancerous gastric lesions. J Natl Cancer Inst 2006;98:974-83.

51. Sung JJ, Lin SR, Ching JY, et al. Atrophy and intestinal metaplasia one year after cure of $\mathrm{H}$. pylori infection: a prospective, randomized study. Gastroenterology 2000;119:7-14.

52. Leung WK, Lin S-R, Ching JYL, et al. Factors predicting progression of gastric intestinal metaplasia: results of a randomised trial on Helicobacter pylori eradication. Gut 2004;53:1244-9.

53. Zhou L, Sung JJY, Lin S, et al. A five-year follow-up study on the pathological changes of gastric mucosa after $\mathrm{H}$. pylori eradication. Chin Med J 2003;116:11-14.

54. Zhou L, Lin S, Ding S. Eight-Year follow-up study on prevalence of gastric cancer and the histopathological changes of gastric mucosa after $\mathrm{H}$ pylori eradication. Chinese Journal of Digestion 2005;25:324-7.

55. Zhou L, Lin S, Ding S, et al. Relationship of Helicobacter pylori eradication with gastric cancer and gastric mucosal histological changes: a 10-year follow-up study. Chin Med J 2014;127:1454-8.

56. Wong BCY, Zhang L, Ma J-ling, et al. Effects of selective COX-2 inhibitor and Helicobacter pylori eradication on precancerous gastric lesions. Gut 2012;61:812-8.

57. Saito D, Boku N, Fujioka T, et al. Impact of H-pylori eradication on gastric atrophy: current status of the Japanese intervention trial (JITHP study). Proceedings of the 6th International Gastric Cancer Congress, 2005:131-6.

58. Correa P, Fontham ET, Bravo JC, et al. Chemoprevention of gastric dysplasia: randomized trial of antioxidant supplements and antiHelicobacter pylori therapy. J Natl Cancer Inst 2000;92:1881-8.

59. Zhou L-ya, Shen Z-yao, Lin S-ren, et al. [Changes of gastric mucosa histopathology after Helicobacter pylori eradication]. Zhonghua Nei Ke Za Zhi 2003;42:162-4.

60. Saito D. [H. pylori eradication for preventing the development and progression of gastic mucosal atrophy: the Japanese Intervention Trial of H. Pylori (JITHP study)]. Helicobacter Research 2006;10:538-42.

61. Tang J, Chen Y, Loke Y, et al. The effects of Helicobacter pylori eradication on histological changes of gastric mucosa and gastric cancer incidence: a three-year follow-up study]. Chinese. Modern Digestion \& Intervention 2010;15:47-9.

62. Mera R, Fontham ETH, Bravo LE, et al. Long term follow up of patients treated for Helicobacter pylori infection. Gut 2005;54:1536-40.

63. Mera RM, Bravo LE, Camargo MC, et al. Dynamics of Helicobacter pylori infection as a determinant of progression of gastric precancerous lesions: 16-year follow-up of an eradication trial. Gut 2018;67:1239-46.

64. Seta T, Takahashi Y, Noguchi Y, et al. Effectiveness of Helicobacter pylori eradication in the prevention of primary gastric cancer in healthy asymptomatic people: a systematic review and metaanalysis comparing risk ratio with risk difference. PLoS One 2017;12:e0183321.

65. Menon S, Trudgill N. How commonly is upper gastrointestinal cancer missed at endoscopy? A meta-analysis. Endosc Int Open 2014;02:E46-E50. 